\title{
Off-Grid Sparse SAR Image Reconstruction by EMMP Algorithm
}

\author{
Salih Uḡur \\ Meteksan Savunma A.S. \\ Ankara, Turkey \\ Bilkent University \\ Electrical and Electronics \\ Engineering Department \\ Ankara, Turkey
}

\author{
Orhan Arıkan \\ Bilkent University \\ Electrical and Electronics \\ Engineering Department \\ Ankara, Turkey
}

\author{
A. Cafer Gürbüz \\ TOBB University of Economics \\ and Technology \\ Electrical and Electronics \\ Engineering Department \\ Ankara, Turkey
}

\begin{abstract}
A new and robust sparse SAR image reconstruction technique is proposed for off-grid targets in the CS framework. In the proposed approach, basis vectors corresponding to ongrid point reflectors are perturbed on a finer grid to find the appropriate bases for the reconstruction of off-grid targets. To provide efficiency of the reconstruction, the EMMP algorithm is applied to find reflectivity center locations. As demonstrated by simulations, the proposed approach significantly improves the performance of sparse SAR image reconstruction.
\end{abstract}

\section{INTRODUCTION}

SAR (Synthetic Aperture Radar) is an active sensor providing high resolution maps of ground reflectance. A platform carrying the SAR system travels through a path while the radar transmits RF pulses towards the scene of interest. The reflected waves are coherently captured throughout the flight path of the SAR platform which constitutes the synthetic aperture.

The received SAR signal at contiguous positions on the flight path which are called as "slow time samples" is discretized by an A/D converter providing "fast time samples". Hence, slow and fast time samples constitute two-dimensional raw SAR data. By using the received signal on a discrete grid, classical SAR image formation processes provide SAR images with pixels corresponding only on-grid reflectivity centers. But reflectivity centers of targets generally are not located at exactly on-grid points as illustrated by Fig. 1. Therefore the energy of an off-grid located target reflectivity center is distributed among the neighboring on-grid pixels which causes loss of contrast as well as false target detections.

Compressed Sensing (CS) is a technique [1] that can provide sparse signal reconstructions even when the signal is sampled well below its corresponding Nyquist rate. It is applied successfully to radar and SAR signal processing area [2], [3], [4]. By carefully designing the sampling process, CS based radar helps reduce required memory size and A/D converter rate [5] of signal processor, resulting important cost and complexity savings. Like classical SAR image reconstruction techniques, CS SAR applications generally do not consider off-grid effects and construct the measurement matrix only by using the basis vectors corresponding to on-grid locations. Unlike classical SAR reconstruction, CS SAR techniques suffer unacceptable

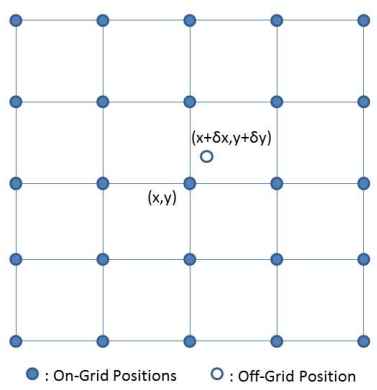

Fig. 1. Off-Grid and On-Grid Points on a Discrete Grid.

degradation in image quality in the presence of off-grid reflectivity centers. This problem is addressed in a recent work on CS SAR image reconstruction [6]. Also, a similar approach including iterative refinement of the dictionary elements is proposed for the problem of parameter estimation for signals characterized by sums of parametrized functions [7].

A new technique called Expectation Maximization Matching Pursuit (EMMP) algorithm [8] has been applied to reconstruct sparse SAR images with unknown phase errors [9]. The EMMP algorithm is greedy and computationally less complex resulting fast SAR image reconstructions. In EMMP algorithm, measurements are considered as incomplete and expectation/maximization (EM) steps are applied iteratively to obtain the complete data.

In our previous SAR imaging technique [9], the measurement matrix, relating measurements to unknown reflectivities, is formed by basis vectors corresponding to on-grid reflectivity centers. To take into account off-grid case in EMMP algorithm based CS SAR image reconstruction, the measurement matrix should cover the basis vectors corresponding to off-grid points. The proposed technique perturbs on-grid basis vectors to match off-grid reflectivity responses.

In the proposed technique, the target is modeled as containing multiple reflectivity points which can be located on off- 
grid points. The proposed technique aims to find the off-grid reflectivity point estimates of a target. If there exist closely located multiple targets in the imaged scene, the proposed technique can be applied to all targets hierarchically one by one.

The formulation of the received SAR signal in CS framework is given in Section Two. Section Three details the offgrid SAR image reconstruction technique. The performance of the proposed technique will be investigated by simulations in Section Four. Section Five contains conclusions.

\section{CS SAR SIGNAL MODEL}

In spotlight mode of operation, the SAR system transmits electromagnetic signals and receives their reflected echoes from the scene along a straight flight path while pointing the antenna beam axis towards the scene center. In this mode, the relation between the received signal vector and the reflectivity vector can be written as [2],

$$
\mathbf{y}=\mathbf{G} \mathbf{x}+\mathbf{w},
$$

where $\mathbf{y}$ is the total received signal (the measurement vector) through the synthetic aperture, $\mathbf{G}$ is the complex valued discrete SAR projection operator matrix, $\mathbf{x}$ is the reflectivity vector and $\mathbf{w}$ is the additive complex white Gaussian measurement noise vector. Assuming that $\mathbf{x}$ is sparse in the column space of a known matrix $\Psi$ with representation coefficients $\alpha$, measurements can be rewritten as:

$$
\mathbf{y}=\mathbf{G} \boldsymbol{\Psi} \alpha+\mathbf{w}=\mathbf{A} \alpha+\mathbf{w} .
$$

By using CS techniques, SAR image reconstruction can be formulated as a Basis Pursuit Denoising (BPDN) problem,

$$
\min _{\alpha}\|\alpha\|_{1} \text { s.t. }\|\mathbf{y}-\mathbf{A} \alpha\|_{2} \leq \sigma,
$$

where the scene with minimum $l_{1}$ norm is reconstructed such that the resulting fit error to measurements is less than a given threshold $\sigma$. A similar formulation which is known as the LASSO can also be written,

$$
\min _{\alpha}\|\mathbf{y}-\mathbf{A} \alpha\|_{2} \quad \text { s.t. }\|\alpha\|_{1} \leq \tau,
$$

where the scene with a known $l_{1}$ norm $\tau$, is chosen to minimize the fit error. These formulations are equivalent for a properly chosen $(\sigma, \tau)$ pair, but it is not straightforward to determine $\sigma$ for SAR image reconstructions especially if the terrain reflectivity is highly variable. However, an appropriate choice for $\tau$ can be obtained based on the size and reflectivity of the dominant reflectors in the imaged area. Hence, it is easier to choose a proper $\tau$, for the $l_{1}$-norm of the target.

\section{OFF-GRID SAR RECONSTRUCTION BY EMMP ALGORITHM}

CS based optimizations aim to obtain the most sparse signal representation, which corresponds to $l_{0}$ norm minimization [1]. But the application of $l_{0}$ norm minimization generally is not feasible, because it requires combinatoric search which results prohibitive computational load. Instead CS optimizations are formulated as $l_{1}$ norm minimizations as in Eqs. 3 and 4 whose solutions require lesser computational loads. The solutions obtained by $l_{0}$ and $l_{1}$ norm minimizations are proved to be equivalent if $\alpha$ is sparse and matrix $A$ holds RIP [10].

Contrary to BPDN and LASSO formulations, the EMMP approach provides a near optimal solution to the following $l_{0}$ norm problem:

$$
\min _{\alpha}\|\mathbf{y}-\mathbf{A} \alpha\|_{2} \quad \text { s.t. }\|\alpha\|_{0} \leq K
$$

where $K$ is the sparsity level of the signal. Like $\tau$ parameter in LASSO formulation, $K$ can be estimated for man-made targets based on the area of these targets in the scene. The EMMP algorithm is recently proposed and is used for an efficient, fast and near optimal solution of Eq. 5. By using the expectation maximization [11] (EM) framework, the EMMP algorithm operates between two spaces: the incomplete and complete data spaces. The incomplete data space corresponds to SAR measurements $\mathbf{y}$, and the complete data space is the set of measurement vectors $\mathbf{y}_{i}$ 's that correspond to the contribution of a single scatterer in the scene. The mapping from the complete data to the incomplete data space is:

$$
\mathbf{y}=\left[\mathbf{A}_{1} \mathbf{A}_{2} \ldots \mathbf{A}_{N}\right]\left[\alpha_{1} \alpha_{2} \ldots \alpha_{N}\right]^{T}=\sum_{i=1}^{N} \alpha_{i} \mathbf{A}_{i} .
$$

Assuming that $\alpha$ has a $K$ sparse representation meaning that only $K$ of $\alpha_{i}$ 's are nonzero, the measurements can be written as,

$$
\mathbf{y}=\sum_{i=1}^{K} \mathbf{y}_{i}+\mathbf{w}
$$

The basics of EMMP algorithm are summarized in Table I. In EMMP iterations, given an estimate for $\mathbf{y}_{i}$, the $i^{\text {th }}$ sparse component of $\alpha$ is found as the best matching vector among the columns of $\mathbf{A}$ to $\mathbf{y}_{i}$, for $1 \leq i \leq K$. Measurement matrix, measurement vector, sparsity level and termination criteria threshold are the inputs of the algorithm. Initially, complete data matrix is set to zero and residual vector is set to measurement vector. Then, expectation and maximization steps are calculated until the termination criteria is met. The termination criteria threshold $\epsilon$, can be set to the average energy of background pixels around the region of interest. At every step, residual vector is also updated. As an output, the algorithm results the reflectivity vector $\mathbf{x}$.

The proposed technique based on the EMMP algorithm is summarized in Table II. First it finds the on-grid point reflectors by calling the EMMP algorithm. The sparsity level, $K$ is adjusted according to the target to be imaged. The result of the first run of the EMMP algorithm is the on-grid target position estimates. Together with estimated on-grid points, the corresponding basis vectors are also found as given in Eq. 6. In order to adjust for actual reflectivity centers which can be located on off-grid points, the basis vectors, $\mathbf{A}_{i}$ 's, are perturbed around the neighborhood of the estimated on-grid point. The perturbation is handled by constructing a finer grid around the estimated on-grid point as illustrated by Fig. 2. 
TABLE I

EMMP ALGORITHM

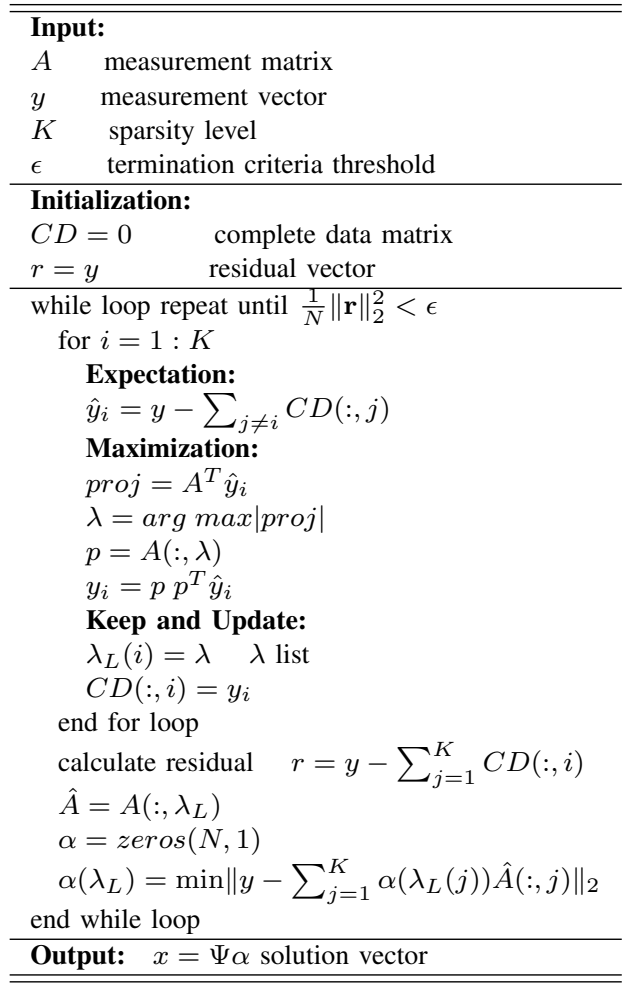

TABLE II

THE PRoposed SAR-EMMP ALGORITHM

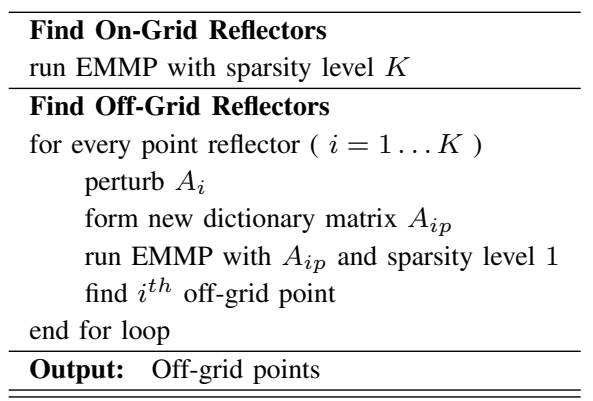

These finer grid points generate basis vectors forming a new dictionary matrix. Therefore, for every on-grid position, a new dictionary matrix $\mathbf{A}_{i p}$ is formed from the small set of perturbed basis vectors $\mathbf{A}_{i p_{j}}$. Then, the EMMP algorithm is run with this new dictionary matrix, $\mathbf{A}_{i p}$ and with the sparsity level set to 1 . This time the algorithm provides estimates on a finer grid for the off-grid point around the on-grid point $i$. These steps are repeated for all on-grid points resulting all the corresponding off-grid point estimates on a finer grid. If the resulted resolution is considered inadequate, then the steps of the algorithm can be repeated hierarchically for even finer grids resulting higher resolutions for the off-grid points.

\section{Simulations}

A synthetically generated SAR data is used to illustrate the performance of the proposed technique. The measurement data

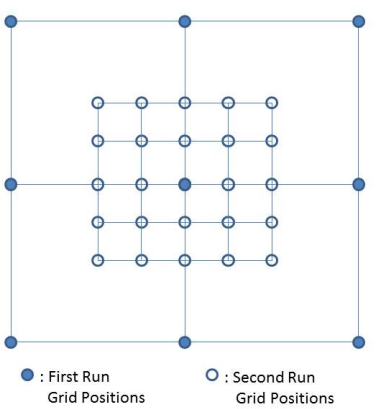

Fig. 2. Grid points for the reconstruction of the basis vectors. For the first run of the EMMP algorithm the points with full circles are used to reconstruct the basis vectors. The first run gives the on-grid point estimates. Around that point, resolution is increased by constructing a finer grid. Hence, for the second run of the EMMP algorithm the points with empty circles are used to reconstruct the basis vectors.

is undersampled randomly by the method explained in [5], so only $40 \%$ of the original raw data is used in the reconstruction by the proposed technique. The identity matrix is used as the sparsity transform because the image domain is sparse for the synthetic data. The azimuth and range coordinates of the off-grid target reflectivity centers are given in the Actual Coordinates column of Table III.

TABLE III

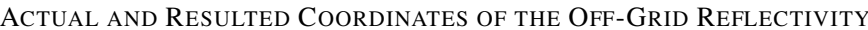
CENTERs.

\begin{tabular}{|c|c|c|c|c|}
\hline \multirow{2}{*}{$\begin{array}{c}\text { Point } \\
\text { No }\end{array}$} & \multicolumn{2}{|c|}{ Actual Coordinates (m) } & \multicolumn{2}{c|}{ Output Coordinates (m) } \\
\cline { 2 - 5 } & Azimuth & Range & Azimuth & Range \\
\hline $\mathbf{1}$ & 0.342 & 0.114 & 0.3 & 0.1 \\
\hline $\mathbf{2}$ & 2.133 & 2.215 & 2.1 & 2.2 \\
\hline $\mathbf{3}$ & -2.267 & 2.283 & -2.3 & 2.3 \\
\hline $\mathbf{4}$ & 2.221 & -2.119 & 2.2 & -2.1 \\
\hline $\mathbf{5}$ & -2.313 & -2.225 & -2.3 & -2.2 \\
\hline
\end{tabular}

The image reconstructed by the classical PFA (Polar Format Algorithm) [13] is given in Fig. 3. Due to off-grid target points, the image is blurred and the energy of the off-grid points spread to the neighboring on-grid points.

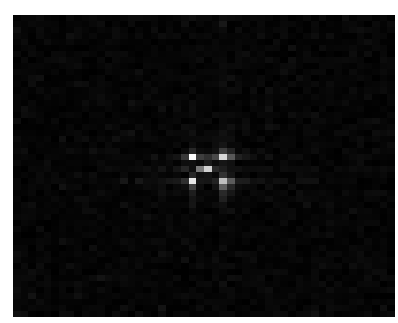

Fig. 3. The image reconstructed by PFA. The pixel resolution is $1 \mathrm{~m}$. in both azimuth and range directions.

The image given in Fig. 4 is reconstructed by the EMMP 
algorithm with sparsity parameter $K=5$. The image presents only the neighboring on-grid points with reduced energy and not giving a clue about the off-grid points. Reconstructing the image by the EMMP algorithm with different $K$ parameters reveals the effects of the off-grid points. The effect of $K$ parameter on image reconstruction quality is analyzed in [12]. Fig. 5 displays an output image reconstructed by the EMMP algorithm with $K=10$. The effects of the off-grid points are clearly seen as their energy disperses to the neighboring pixels.

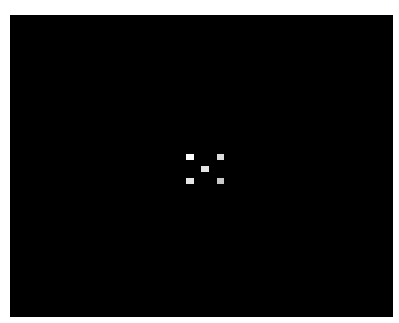

Fig. 4. The image reconstructed by EMMP algorithm with sparsity parameter $K=5$. The pixel resolution is $1 \mathrm{~m}$. in both azimuth and range directions.

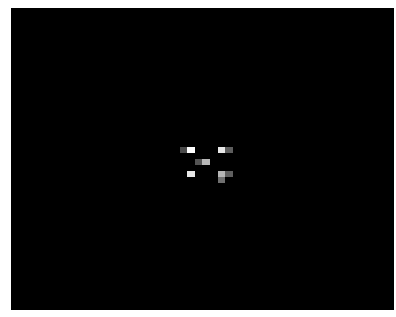

Fig. 5. The image reconstructed by EMMP algorithm with sparsity parameter $K=10$. The pixel resolution is $1 \mathrm{~m}$. in both azimuth and range directions.

The proposed technique based on the iterative application of EMMP algorithm to find the off-grid target points is applied to the synthetically generated data. The technique is applied to the data just one iteration which results finer grid resolution around the on-grid locations. One iteration of the technique resulted accuracy level set to the tenths digit. The results are listed in the Output Coordinates column of Table III. The resulted coordinates obtained by the proposed technique match with the actual coordinates up to tenths digit which proves the efficient application of the method. Because grids become finer in every application and they determine the resolution of the reconstruction, higher resolutions can be obtained by repeated application of the technique to the off-grid points. But this method has a resolution limit reached when the base vectors created by finer grids do not result a residual change greater than $\epsilon$ in EMMP algorithm. The relation between the achievable resolution level of the reconstruction and the parameter $\epsilon$ which is related to the noise level of the measured data will be investigated thoroughly in a future work.
The perturbation of basis vectors around the neighborhood of the on-grid points results the detection of off-grid points with high precision. The EMMP algorithm is a fast one compared to other alternative CS reconstruction techniques like conjugate gradient methods. The proposed technique calls the EMMP algorithm inside a for loop with small sized $A_{i p}$ matrix just for the neighboring off-grid points and with sparsity level $K=1$, which results computational efficiency and speed.

\section{CONCLUSIONS}

The proposed EMMP based technique provides efficient SAR image reconstruction for targets with off-grid reflectivity centers. Because it uses CS framework, the technique requires only a fraction of the Nyquist rate samples. Hence the proposed technique also relaxes the requirements on the SAR hardware. Contrary to alternative $l_{1}$ norm minimization based approaches, the EMMP based technique provides near optimal solution to the desired $l_{0}$ norm minimization problem efficiently. Iterative application of the EMMP algorithm to find the off-grid target points provides fast reconstructions due to the faster convergence of the EMMP algorithm compared to alternative gradient descent based optimization techniques for CS reconstructions. Numerical experiments on the synthetic data set show that the proposed technique provides off-grid coordinates for reconstruction with high precision.

\section{REFERENCES}

[1] D. L. Donoho, “Compressed Sensing," IEEE Transactions on Information Theory, vol. 52, no. 4, pp. 1289-1306, April 2006.

[2] V. M. Patel, G. R. Glenn, D. M. Healy and R. Chellappa, "Compressed Synthetic Aperture Radar," IEEE Journal of Selected Topics in Signal Processing, vol. 4, no. 2, pp. 244-254, April 2010.

[3] M. Herman and T. Strohmer, "Compressed Sensing Radar," IEEE Radar Conference 2008, pp. 1-6, May 2008.

[4] A. C. Gurbuz, J. H. McClellan and W. R. Scott, "A Compressive Sensing Data Acquisition and Imaging Method for Stepped Frequency GPRs, " IEEE Transactions on Signal Processing, vol. 57, iss. 7, pp. 2640-2650, July 2009.

[5] S. Uḡur and O. Arıkan, "SAR Image Reconstruction and Autofocus by Compressed Sensing," Digital Signal Processing, vol. 22, iss. 6, pp. 923-932, December 2012

[6] A. Fannjiang and H.C Tseng, "Compressive Radar with Off-Grid and Extended Targets," arXiv:1209.6399.

[7] C. D. Austin, J. N. Ash and R. L. Moses, "Parameter Estimation Using Sparse Reconstruction With Dynamic Dictionaries," ICASSP 2011, May 2011.

[8] A. C. Gürbüz, M. Pilancı and O. Arıkan, "Expectation Maximization Based Matching Pursuit," ICASSP 2012, March 2012.

[9] S. Uḡur, A. C. Gürbüz and O. Arıkan, "Autofocused Sparse SAR Image Reconstruction by EMMP Algorithm," $1^{\text {st }}$ International Workshop on Compressed Sensing Applied to Radar, 14-16 May 2012, Bonn, Germany.

[10] E. Candès, J. Romberg, and T. Tao, "Stable Signal Recovery from Incomplete and Inaccurate Measurements, "Communications on Pure and Applied Mathematics, vol. 59, no. 8, pp. 1207-1223, 2006.

[11] A. Dempster, N. Laird and D. Rubin, "Maximum Likelihood from Incomplete Data via the EM Algorithm," J. Roy. Statist. Soc. Ser. B., vol. 39, no. 1, pp. 1-38 1977.

[12] S. Uḡur, O. Arıkan and A. C. Gürbüz, "Autofocused Sparse SAR Image Reconstruction by EMMP Algorithm," submitted to IEEE Transactions on Aerospace and Electronic Systems.

[13] D. C. Munson, Jr., J. C O'Brien, and W. K. Jenkins, ”A Tomographic Formulation of spotlight-mode synthetic aperture radar, "Proc. IEEE, vol. 71, no. 8, pp. 917-925, August 1983. 